\title{
ARTICLE
}

\section{Nanopore long-read RNAseq reveals widespread transcriptional variation among the surface receptors of individual $B$ cells}

\author{
Ashley Byrne ${ }^{1,2}$, Anna E. Beaudin ${ }^{3, \dagger}$, Hugh E. Olsen ${ }^{2,3}$, Miten Jain ${ }^{2,3}$, Charles Cole ${ }^{2,3}$, Theron Palmer ${ }^{3}$, \\ Rebecca M. DuBois ${ }^{3}$, E. Camilla Forsberg ${ }^{3,4}$, Mark Akeson ${ }^{2,3}$ \& Christopher Vollmers ${ }^{2,3}$
}

Understanding gene regulation and function requires a genome-wide method capable of capturing both gene expression levels and isoform diversity at the single-cell level. Short-read RNAseq is limited in its ability to resolve complex isoforms because it fails to sequence fulllength cDNA copies of RNA molecules. Here, we investigate whether RNAseq using the longread single-molecule Oxford Nanopore MinION sequencer is able to identify and quantify complex isoforms without sacrificing accurate gene expression quantification. After benchmarking our approach, we analyse individual murine Bla cells using a custom multiplexing strategy. We identify thousands of unannotated transcription start and end sites, as well as hundreds of alternative splicing events in these Bla cells. We also identify hundreds of genes expressed across B1a cells that display multiple complex isoforms, including several B cellspecific surface receptors. Our results show that we can identify and quantify complex isoforms at the single cell level.

\footnotetext{
${ }^{1}$ Department of Molecular, Cell, and Developmental Biology, University of California-Santa Cruz, 1156 High Street, Santa Cruz, California 95064, USA.

2 UC Santa Cruz Genomics Institute, 1156 High Street, Santa Cruz, California 95064, USA. ${ }^{3}$ Department of Biomolecular Engineering, University of CaliforniaSanta Cruz, 1156 High Street, Santa Cruz, California 95064, USA. ${ }^{4}$ Institute for the Biology of Stem Cells, Department of Biomolecular Engineering, University of California-Santa Cruz, 1156 High Street, Santa Cruz, California 95064, USA. † Present address: Department of Molecular and Cell Biology, School of Natural Sciences, University of California-Merced, 5200 Lake Rd, Merced, California 95343, USA. Correspondence and requests for materials should be addressed to C.V. (email: vollmers@ucsc.edu).
} 
O ver the last decade, RNAseq has vastly increased our knowledge of eukaryotic gene expression and the unique transcript isoform signatures that differentiate developmental stages, organs and single cells ${ }^{1-4}$. Proteins that arise from transcript isoforms of a single gene can vary in their biological properties including stability, intracellular localization, enzymatic activity and post-translational modifications ${ }^{5}$. Transcript isoforms are the product of alternative transcription start sites (TSSs), transcription end sites (TESs) and alternative splicing events that include alternative splice sites, intron retention and exon skipping ${ }^{6}$. It has been predicted that a large fraction of human genes is alternatively spliced ${ }^{7,8}$. Although alternative splicing enables increased transcriptome diversity, aberrations in splicing have been implicated in several human diseases, including cancer. Indeed, up to $15 \%$ of all point mutations causing human genetic disease are predicted to result in mRNA splicing 9 somatic mutations within splicing factors are associated with 12 different cancer types ${ }^{10}$.

Consequently, it is important to determine the true transcriptional diversity of cells. This requires that gene expression is analysed not only at the gene-level but also at the isoform-level. However, current short-read RNAseq methods are inherently limited in their ability to identify complex transcript isoforms, as they cannot sequence full-length transcripts. Instead, transcripts are fragmented for sequencing, resulting in short individual reads that fail to span the entirety of the transcript. Computational tools can be used to assemble full-length transcripts from these reads, but different assembly algorithms can result in conflicting outcomes and varying overall assembly quality ${ }^{11}$.

To offset this limitation of short-read RNAseq, studies have successfully used both single-molecule long-read PacBio Iso-Seq and synthetic long-read MOLECULO methodologies ${ }^{12-15}$ to sequence full-length complementary DNA (cDNA). Recently, the Oxford Nanopore Technologies (ONT) MinION has been used to analyse full-length cDNA samples derived from both defined synthetic RNA molecules, as well as RNA from tissue culture cells $^{16,17}$.

With only two exceptions ${ }^{18,19}$, these long-read technologies have been used exclusively to evaluate transcriptome diversity across bulk cell populations. However, recent studies have highlighted that cells found within seemingly homogeneous populations can differ in gene expression ${ }^{20-22}$. Understanding heterogeneity within cell populations has shown promise across multiple disciplines including developmental biology, neurobiology, cancer and immunology. Single-cell approaches can help illuminate biological questions regarding cell function, development and dysfunction. Knowing the exact state of the cell can help determine its fate or reflect changes with response to stimuli or drug treatment, as well as its ability to neutralize a pathogen, respectively. Cell-to-cell heterogeneity ${ }^{3}$ makes immune cells a fascinating target for in-depth analysis of transcriptional diversity. Current approaches that measure RNA transcripts within single cells rely on short-read RNA-seq, single-molecule RNA-fluorescence in-situ Hybridization (SM-RNA FISH) or single-cell RT-qPCR ${ }^{23-26}$. These current methods can either be applied to a few genes or are under the same constraints of shortread RNA-seq, which we described earlier. Ultimately, these approaches are limited in their ability to identify and quantify complex isoforms, containing multiple, possibly distant, alternative events, on a transcriptome-wide level.

To make it possible to identify and quantify complex isoforms on a transcriptome-wide single-cell level, we have developed a nanopore sequencing approach for the analysis of full-length cDNA in single cells. The ONT MinION sequencer is a portable device that is based on single-molecule sequencing technology that provides reads of unprecedented length by performing voltage-driven molecule translocations through small nanosensors $^{27}$. Although the MinION platform has been most useful for interrogating viral and bacterial genomes, recently it has been applied for analysing cDNA in both targeted, as well as genomewide approaches $16,28-31$.

Taking advantage of its unprecedented read length, we wanted to interrogate single-cell transcriptomes of mouse B1a cells by sequencing full-length cDNA molecules using the ONT MinION sequencer. We implemented an integrated informatics pipeline (Mandalorion) for gene-level and transcript isoform-level expression quantification to overcome the sequencing accuracy limitations of the ONT MinION. To identify transcript isoforms, Mandalorion predicted transcription start and end sites, as well as splice sites and their alternative usage. After benchmarking the ONT RNAseq approach on a complex mixture of synthetic transcripts, we sequenced seven individual mouse B1a cells to show that we could accurately quantify gene expression and identify and quantify novel isoforms at the single-cell level. Our analysis identifies differential usage of complex isoforms in over a hundred genes including several surface molecules like CD19, $C D 20$ and $I G H$, the very receptors defining B cell identity.

\section{Results}

Generating and sequencing single-cell RNAseq libraries. We first investigated the ability of the ONT MinION platform to interrogate transcriptomes at the single-cell level. To test this, we used our ONT RNAseq approach to analyse seven individual mouse B1a cells ${ }^{32,33}$ and compared it with the standard Illumina RNAseq approach. To this end, we FACS-sorted single B1a cells into individual wells containing lysis buffer and amplified cDNA from each individual cell using the Smartseq2 protocol with modifications (see Methods, Supplementary Table 1) ${ }^{34}$. The cDNA generated by the Smartseq2 protocol was split and processed in-parallel using the Illumina and ONT library preparation protocols. Sequencing the fragmented cDNA of the seven cells on the HiSeq2500, we generated between 73,086 and $351,876150 \mathrm{bp}$ Illumina reads per cell. Sequencing the full-length cDNA of the first three cells on individual ONT MinION flow cells using the R7.3 chemistry generated between 17,749 and 52,696 ONT 2D reads per cell (Supplementary Table 2). Taking advantage of the improved MinION throughput using the R9.4 chemistry, we multiplexed the full-length cDNA of the other four cells on a single MinION flow cell and generated between 57,874 and 128,726 ONT $2 \mathrm{D}$ reads per cell. To enable this multiplexing we introduced custom 60 nucleotide cellular indexes during PCR amplification (see Methods, Supplementary Table 1, Fig. 1a).

Comparison of gene expression quantification. To assess whether ONT RNAseq is capable of quantifying gene expression, we compared RNAseq data produced with ONT and Illumina, the current benchmark for gene expression quantification. Because standard gene quantification tools (for example, STAR ${ }^{35}$, Cufflinks ${ }^{36}$ ) are not compatible with nanopore reads, we aligned the ONT $2 \mathrm{D}$ reads using BLAT $^{37}$ and quantified gene expression using our own Mandalorion pipeline. Mandalorion determines how many reads overlap with the exons of a gene to produce a Reads Per Gene per $10 \mathrm{~K}$ reads (RPG10K) value. As ONT 2D reads are long enough to span the full-length of the transcripts, normalization for gene length was not performed (Fig. 1b). Comparing Illumina and ONT RNAseq gene expression quantification for the same cell showed high correlation (Pearson $r \geq 0.84-0.89$ for R7.3 and 0.9-0.92 for R9.4), confirming that our ONT RNAseq approach recapitulates Illumina gene expression quantification (Fig. 2). Comparing Illumina and ONT RNAseq gene expression quantification across different cells showed low 
a

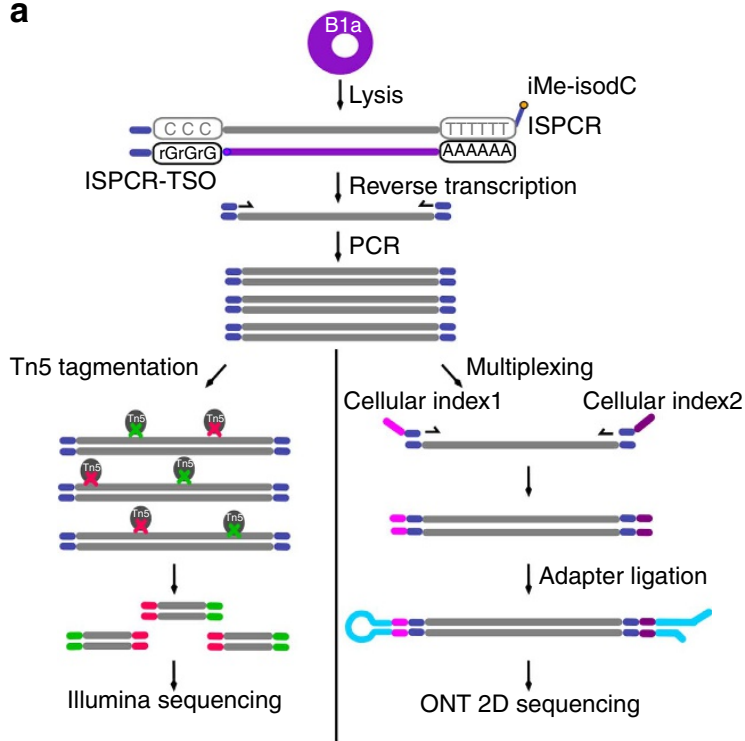

b

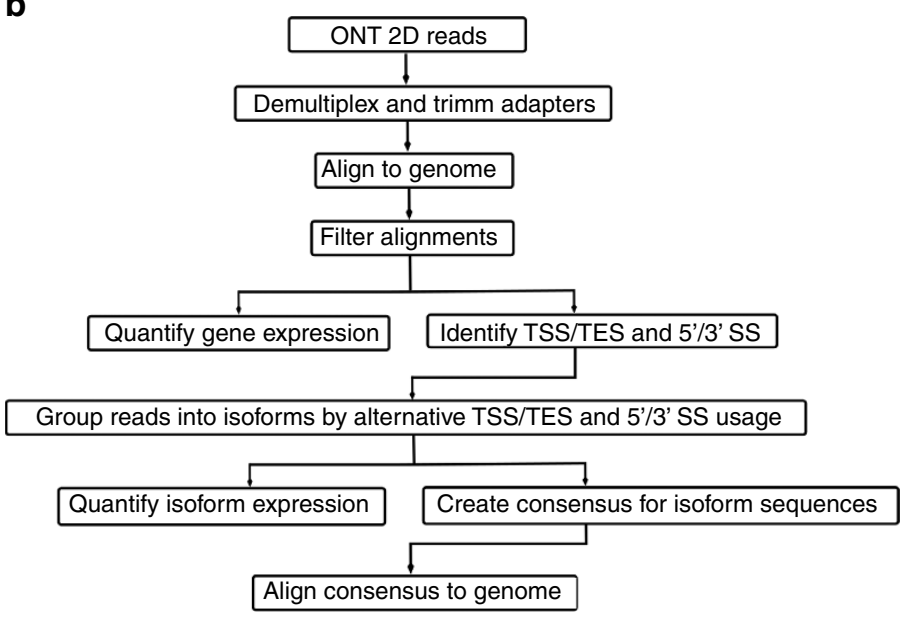

Figure 1 | Experimental design and Oxford Nanopore sequencing read characteristics. (a) Schematic of experimental design. FACS-sorted single B1a cells were lysed. PolyA-RNA was then reverse transcribed and PCR amplified using template switching. Full-length cDNA was split into two reactions. Half of the reaction was tagmented by Tn5 and sequenced using a Illumina HiSeq2500 sequencer. The other half of the reaction was ligated to ONT adaptors and sequenced on an ONT MinION sequencer. (b) Schematic of the Mandalorion pipeline used to analyse the ONT 2D read data.

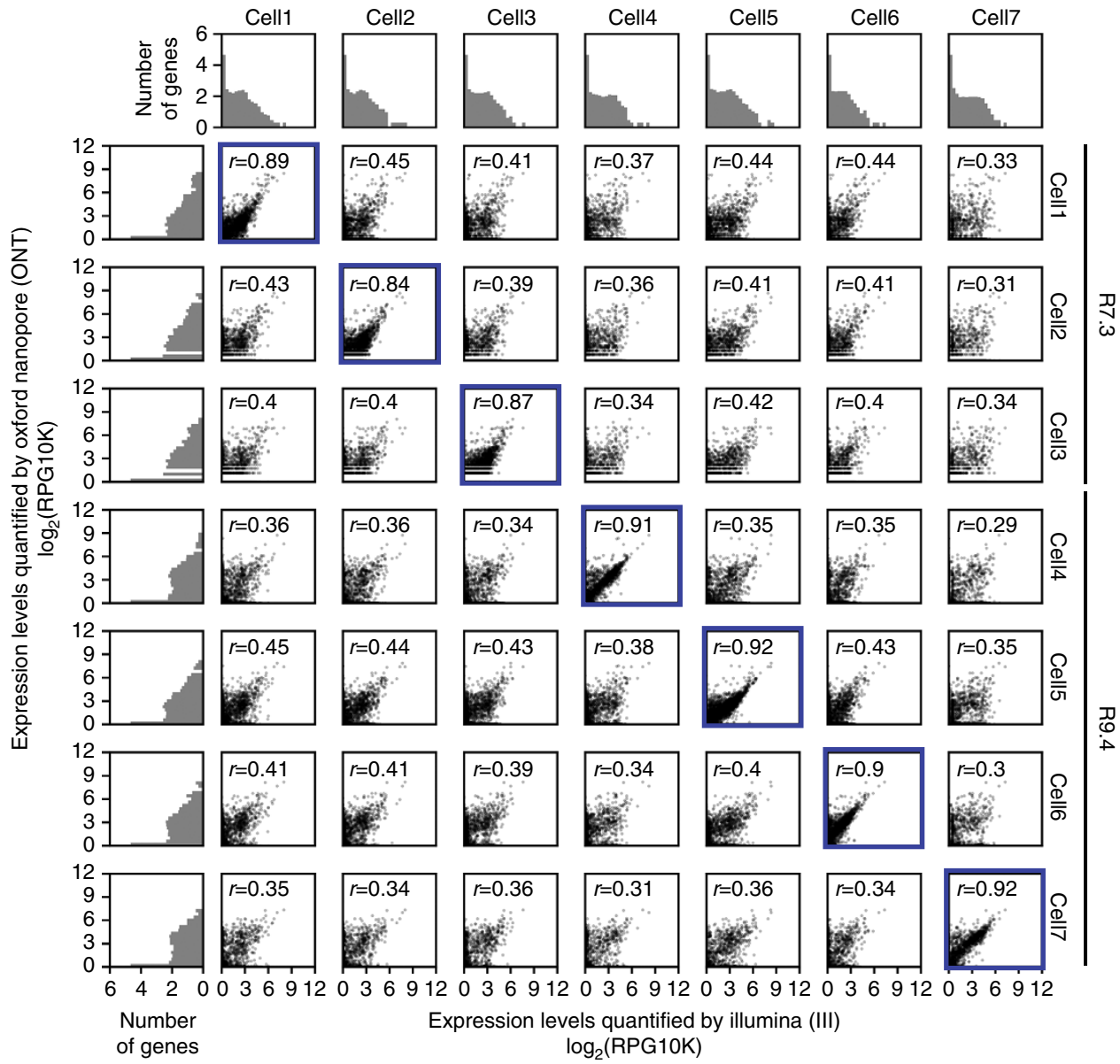

Figure 2 | ONT RNAseq recapitulates Illumina RNAseq gene expression quantification. Scatter plot grid at the centre of the figure shows gene expression levels for each gene as determined by Illumina RNAseq and ONT RNAseq for the indicated cells. Correlations of gene expression levels are given as reads per gene per 10,000 reads (RPG10K) across seven single cells. Pearson $r$ is given for each cell per sequencing method combination with each point representing transcript expression level $(x$-axes = Illumina and $y$-axes=ONT). Same cell comparisons have a blue border. ONT sequencing chemistry is shown on the right. Histograms found on the left and top of the figure represent number of genes found binned by their expression levels. 
correlation with a Pearson $r \leq 0.45$, suggesting that ONT RNAseq can identify cell-to-cell variability ${ }^{1,38}$ (Fig. 2).

These results show that even with the relative low number of reads produced, ONT RNAseq gene-expression quantification largely detects the same genes as Illumina RNAseq (Fig. 3a). Furthermore, subsampling ONT and Illumina raw reads showed that, for five of the seven cells analysed, the detection of expressed genes had reached saturation (Supplementary Fig. 2). Unsurprisingly, genes that were detected by either ONT or Illumina RNAseq alone were expressed at lower levels, indicating that these genes were expressed at levels close to the detection limits of both technologies (Fig. 3b). We also observed that the genes detected by ONT RNAseq alone were comprised of smaller transcripts (Fig. 3c). In addition, genes that were $<600 \mathrm{bp}$ in length and were detected by both ONT and Illumina RNAseq had relatively lower expression levels in Illumina RNAseq data (Fig. 3d). While this is consistent with smaller transcripts being strongly selected against in the Tn5 based Illumina library prep, we could not exclude that ONT RNAseq might have a bias towards shorter transcripts. To exclude this possibility, we chose to analyse a mix of synthetic transcripts.

Analysis of SIRV synthetic transcript mixtures. To test whether transcript length had an effect on expression levels as measured by ONT RNAseq, we sequenced synthetic Spike-in RNA Variant Control Mixes (SIRVs, Lexogen) of known length, structure and sequence. SIRV transcripts provided in the E2 mix contained 69 transcripts ranging from 191 to $2528 \mathrm{nt}$. In the E2 mix 69 transcripts were present in four groups of varying concentrations containing 19,21, 17 and 12 transcripts in each group, respectively. To test a wide range of possible transcript levels, we amplified (sub-) single-cell amounts (that is, $10 \mathrm{fg}$ and $100 \mathrm{fg}$ ) of the Lexogen SIRV E2 mix in duplicate. This reflected a wide range of possible transcript levels with 8-10,240 molecules of individual SIRV transcripts present before the amplification step. a

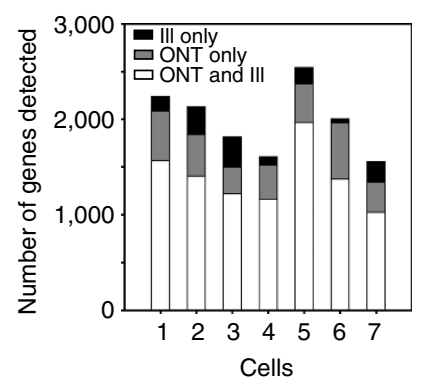

b

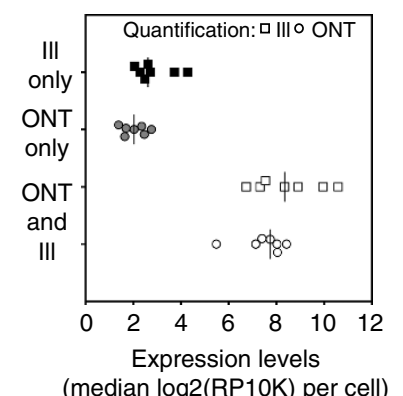

C

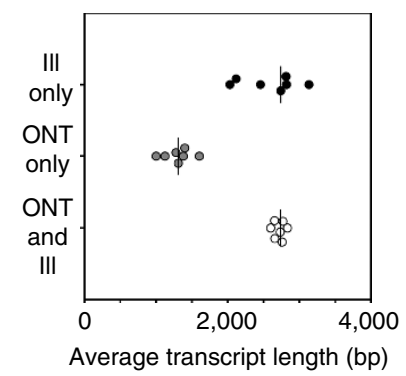

d

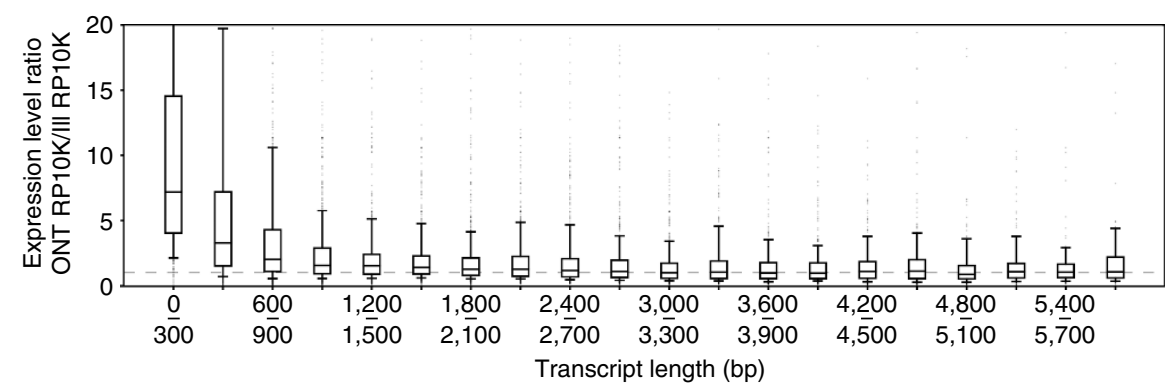

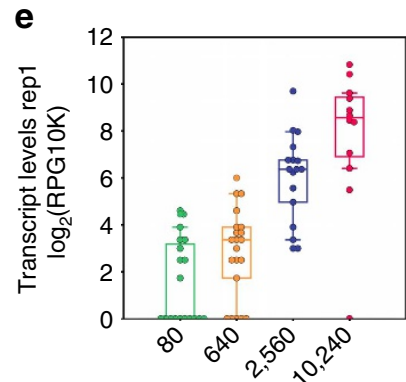

Number of

transcript molecules
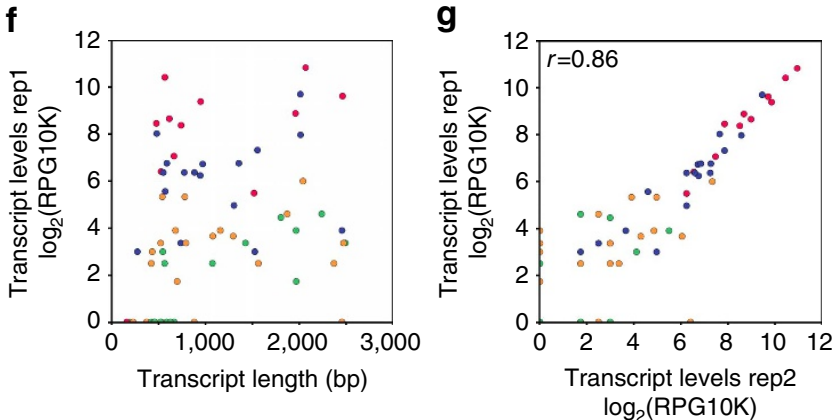

Figure 3 | Quantifying gene and transcript expression with ONT RNAseq data. (a) Stack barplots showing the number of genes detected in each cell corresponding to different sequencing technologies (III-IIlumina, ONT-Oxford Nanopore). (b) Median expression levels of genes detected by both or individual technologies. Two expression levels (III and ONT) are given for genes detected in both technologies. (c) Gene length of genes detected by both or individual technologies. (d) Ratio of gene expression levels for genes detected by both technologies. Ratios are binned according to gene length and shown as boxplots with whiskers indicating 10th and 90th percentiles. (e) SIRV transcript levels of Replicate 1 (Rep1: 100fg SIRV pool E2) as measured with ONT RNAseq. Transcripts are binned by their starting molecule numbers. (f) SIRV transcript levels of Replicate 1 are plotted against transcript length with colours corresponding to groups in e. (g) Scatter plot showing correlation of SIRV transcript expression levels of Replicate 1 (Rep1: 100fg SIRV pool E2) and Replicate 2 (Rep2: $100 \mathrm{fg}$ SIRV pool E2), both measured by ONT RNAseq. $r$-value shown is Pearson $r$. Colours corresponding to groups in e. 
We quantified the 69 transcripts by aligning the resulting 5367-17915 2D ONT reads directly to the spliced SIRV transcriptome using BLAT and then counting and normalizing the matched ONT 2D reads for each transcript. As expected when amplifying (sub-) single-cell amounts of RNA, we observed transcript drop-out in the lower concentration groups and found that transcript quantification showed variations within each concentration group (Fig. 3e, Supplementary Fig. 3a). Most importantly, however, quantification was not affected by transcript length, with the exception of transcripts shorter than $500 \mathrm{bp}$. These transcripts were either underrepresented or missed entirely (Fig. 3f). Generally, ONT RNAseq quantification agreed with the nominal concentration of the spike-in transcripts and, interestingly, the intra-group variations in transcript quantification were reproducible between replicates (Fig. $3 \mathrm{~g}$ ). This intragroup variation might be due to variation in initial transcript levels, systematic amplification bias, or data analysis bias. Overall, the observed underrepresentation of short transcripts in ONT RNAseq and the differences between Illumina and ONT RNAseq quantification are consistent with cDNA molecules below $500 \mathrm{bp}$ in length being selected against during cDNA synthesis and again during the Illumina library preparation using the Tn5 method. Ultimately, analysing these synthetic transcripts at different concentrations allowed us to exclude the possibility that ONT RNAseq favors shorter transcripts.

Next, we wanted to test whether, in addition to largely unbiased quantification of SIRV transcripts $500-2,500 \mathrm{bp}$ in length, ONT RNAseq reads cover transcripts in their entirety which would make them uniquely suitable to identify and quantify complex isoforms.

Isoform identification and quantification from SIRV data. The 69 synthetic SIRV transcripts are derived from seven artificial gene loci that have been modelled after human genes with high isoform diversity, making them suitable for testing ONT RNAseq's capability to capture isoform diversity in a genome annotation independent manner. To this end, we used Mandalorion to analyse ONT RNAseq 2D read data to annotate the SIRV gene loci, which in turn could be utilized to further identify and quantify SIRV isoforms. First, we used read alignments to annotate TSS and TES, as well as splice sites (SS) of SIRV transcripts in the SIRV gene loci. The annotation of TSS and TES was accomplished by end to end coverage of the entire RNA transcript by complete ONT 2D reads (that is, reads for which both ISPCR adaptors could be identified and trimmed, Supplementary Table 2) (Fig. $4 \mathrm{a}-\mathrm{c}$ ). Complete ONT 2D reads contained information regarding both TSS, TES in their read alignments.

After combining and aligning ONT 2D reads of all replicates to the artificial SIRV genomic loci (Fig. 4c, Supplementary Fig. S4), we categorized $20 \mathrm{bp}$ bins containing TSS, TESs and splice sites using the Mandalorion pipeline (see Methods). To avoid the detection of spurious TSS and TES by prematurely terminated read alignments, we required TSS/TES to be at least $60 \mathrm{bp}$ apart. In this manner, we detected 20 TSS and 24 TES that all directly overlapped with an actual TSS and TES and were within $60 \mathrm{bp}$ of 38 (of 57) actual TSSs and 41 (of 59) actual TESs present in the SIRV transcript annotation. Furthermore, we detected 76 (of 89) $5^{\prime}$ splice sites and 73 (of 93) $3^{\prime}$ splice sites present in the SIRV genome annotation. By analysing the actual splicing pattern of ONT 2D reads we detected 11 (of 12) alternative $3^{\prime}$ splice site combinations and 12 (of 14) alternative $5^{\prime}$ splice site combinations, as well as 12 (of 12) intron retention events present in the SIRV transcripts.

Using Mandalorion, we then sorted ONT 2D reads into isoform groups based on their TSS/TES and alternative splice site usage. We generated consensus sequences of these groups using Partial Ordered Alignment (POA) ${ }^{39}$ and compared these consensus sequences to SIRV transcript sequences using BLAT. All of the 33 consensus sequences we generated matched a SIRV transcript with between 97.8 and $100 \%$ identity (BLAT identity score) and in all cases matched its directionality. Of the resulting 33 consensus sequences, 26 matched one of the 29 SIRV transcripts present in the two highest abundance groups (Fig. 4c, Supplementary Fig. 4). The other seven consensus sequences matched one of the 40 SIRV transcripts in the two low abundance groups. While Mandalorion did not succeed in consistently identifying lower abundance isoforms, the consensus isoform sequences detected were very accurate. We also observed high correlation between the genome annotationindependent transcript isoform quantification by Mandalorion and quantification derived from directly aligning reads to the transcriptome (Fig. 4d) This means that in addition to identifying sequence, structure and directionality of complex isoforms, Mandalorion can also accurately quantify them in a genome annotation independent manner. As a result, we were encouraged to apply this pipeline to our single-cell data.

Identification of isoform features used in single B1a cells. By analysing the ONT $2 \mathrm{D}$ reads generated from the seven B1a cells using Mandalorion, we detected 4234 TSSs and 3883 TESs with only 2476 TSSs and 2448 TESs overlapping with the TSSs or TESs present in the Gencode annotation (vM10) ${ }^{40,41}$ of the mouse genome (Fig. 5a). To determine whether the unannotated TSS and TES we detected were artifacts of our experimental and computational pipelines, we determined their Fantom $5^{42}$ CAGE peak and polyA signal enrichment. Fantom5 CAGE peaks are derived from capturing and sequencing the $5^{\prime}$ end of transcripts and should therefore be enriched in TSSs. Indeed, we found that in contrast to TESs (49/3883 or $1.3 \%)$, a high percentage of both annotated $(2356 / 2476$ or $95 \%)$ and unannotated $(1052 / 1799$ or 58\%) TSSs overlapped with high scoring Fantom5 CAGE peaks (Fig. 5b). Conversely, both annotated and unannotated TESs were highly enriched for polyA signals, while TSSs were not (Fig. 5c). When we assigned the detected TSSs and TESs to annotated genes, we found that most genes contained exactly one TSS and one TES, as expected. However, 696 genes contained more than one TSS or TES indicating the presence of more than one isoform (Fig. 5d). Overall, this suggested that Mandalorion successfully identified thousands of unannotated TSSs and TESs and hundreds of genes with differential TSS/TES usage by analysing individual cells.

In addition to TSSs and TESs, Mandalorion identified a total of $24,8875^{\prime}$ splice sites and 24,756 $3^{\prime}$ splice sites. The vast majority of these splice sites were supported by the GENCODE annotation or splice junctions found in Illumina reads. Of the $24,8875^{\prime} \mathrm{SS}$ and 24,756 $3^{\prime}$ SS we identified, 24,298 (97.6\%) and 24,220 (97.8\%) matched GENCODE annotation, respectively. Of the $5895^{\prime} \mathrm{SS}$ and $5363^{\prime}$ SS that did not match GENCODE annotation, 250 (42.4\%) and $216(40.2 \%)$ were supported by splice junctions in Illumina reads, respectively. Even if all splice sites that were not supported by GENCODE annotation or Illumina reads were false, which is unlikely, the false discovery rate of our approach would only be $1.3 \%(659 / 49,643)$. Furthermore, while Mandalorion defined our splice sites as 20 bp bins, we were relatively successful in defining the exact splice site as shown by the base context of the determined splice sites (Fig. 5e). By determining alternative splice sites, we found 296 intron retention events, 134 alternative $5^{\prime}$ splice sites and 173 alternative $3^{\prime}$ splice site combinations. The majority of these events were also observed in Illumina read data, which supported 216 (of 296) intron retention events, 99 (of 134) 
a

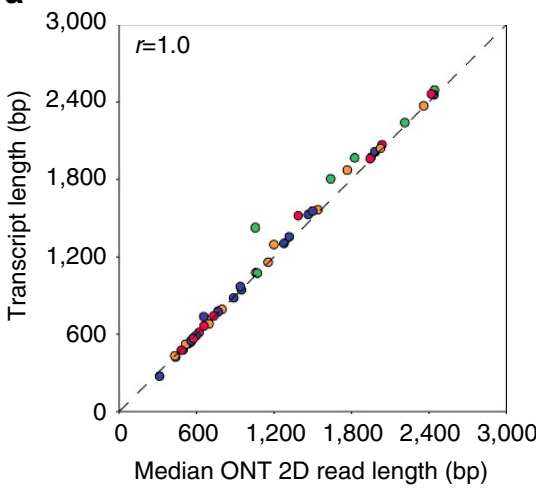

b

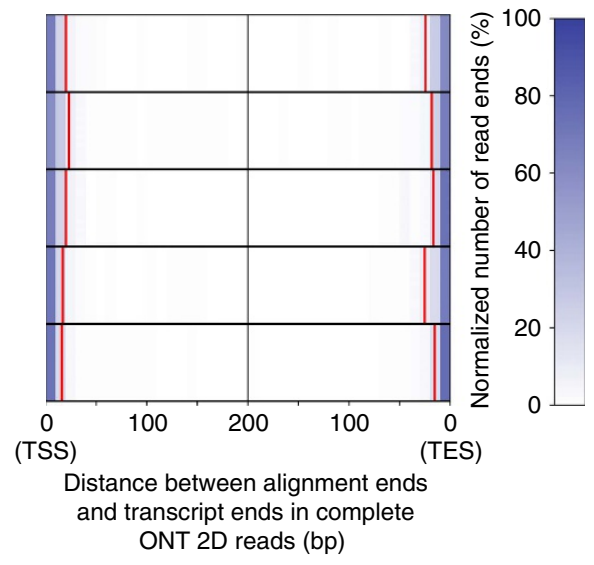

d

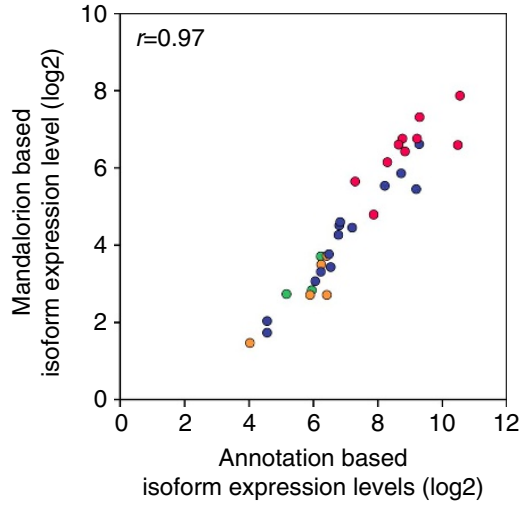

C

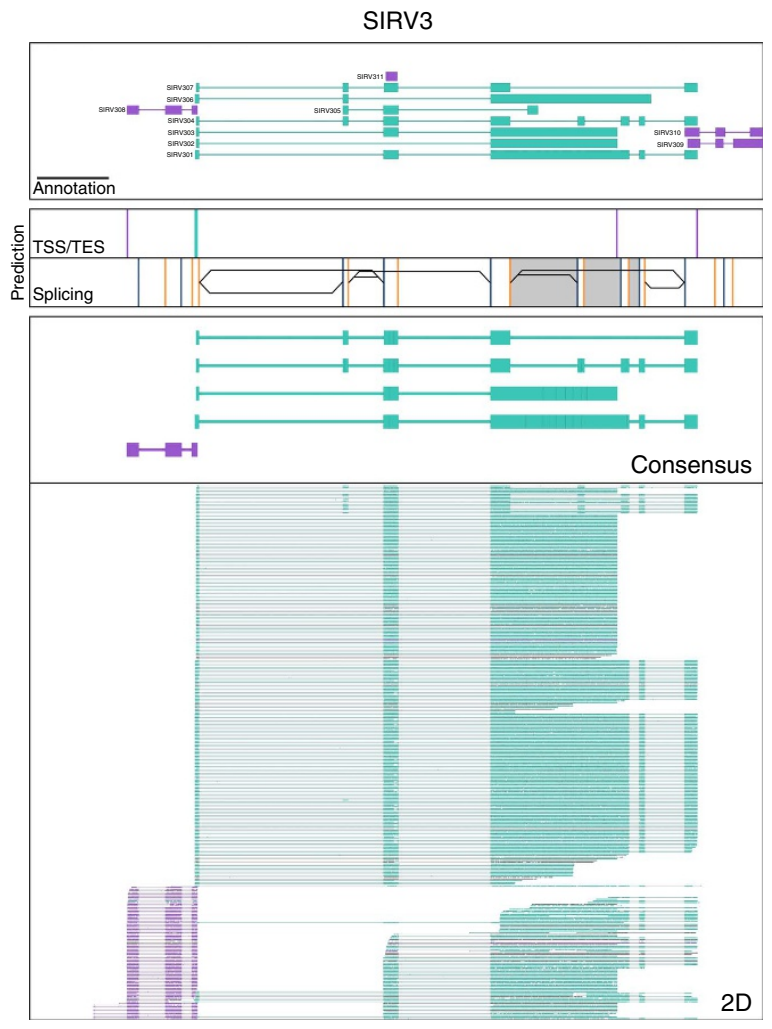

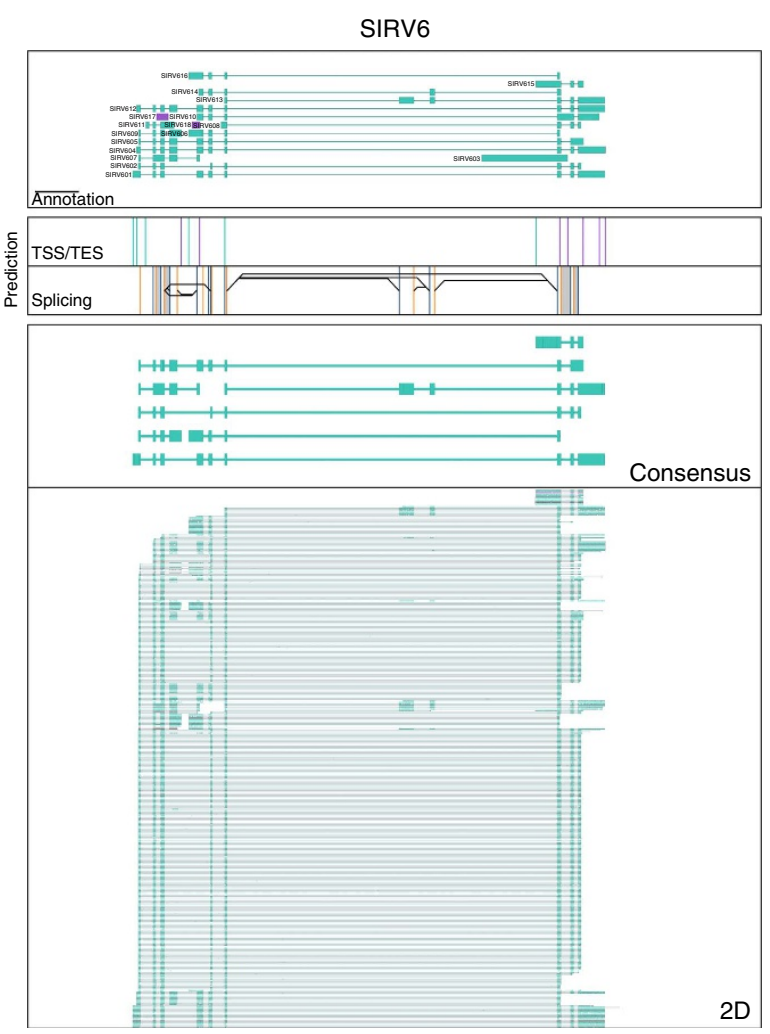

Figure 4 | Identifying and quantifying transcript isoforms in SIRV E2 mixtures. (a) Scatter plot shows correlation between ONT 2D reads and the SIRV transcripts they align to. Pearson $r$ is shown. Colouring is as indicated in Fig. 3e-g (b) Distance between read alignment ends and transcript ends are shown as heatmap with the colour indicating the normalized alignment numbers. $90 \%$ of read alignments terminated outside the red lines (c) Genome Browser view of SIRV3(c) and SIRV6(d) gene loci. Top box contains transcript annotations with black $1 \mathrm{~kb}$ scale bar in bottom left corner, second and third box contain TSS (Teal)/TES (Purple) and splice sites (yellow: 5'SS, blue: $3^{\prime} \mathrm{SS}$ ) locations predicted from the read data, respectively. Black lines and grey areas in box 3 indicate alternative splicing and intron retention events predicted from the read data. Box 4 contains read alignments of isoform consensus reads. Box 5 contains ONT 2D read alignments. Direction of transcripts, isoform consensus, and ONT 2D reads are indicated by their colour (Teal: $5^{\prime}$ to $3^{\prime}$, Purple: $3^{\prime}$ to $5^{\prime}$ ). (d) Scatter plot shows correlation between SIRV transcript quantification by aligning to annotated transcripts or by annotation-independent isoform grouping using Mandalorion. Pearson $r$ is shown.

alternative $5^{\prime}$ splice sites, 123 (of 173) alternative $3^{\prime}$ splice sites and 72 (of 92) exon skipping events (Fig. 5f). Alternative events not supported by Illumina read data had significantly lower ONT $2 \mathrm{D}$ read counts than those that were supported (Supplementary Table S3), indicating they might be closer to the detection limits of both technologies.

Identification of complex isoforms. Having established that ONT RNAseq can be used to identify isoform features like TSSs and TESs as well as alternative splicing events, we aimed to identify complex isoforms. We defined genes as expressing complex isoforms if they contained alternative TSS/TES, as well as alternative splice sites. We identified 169 genes that expressed complex isoforms. By identifying and quantifying all isoforms we detected at these 169 genes, we found highly significant differential isoform usage between cells in 55 of the genes (Chi2contigency test, alpha $=0.001$, holm-sidak multiple-testing correction). These genes with significant differential isoform usage included B cell-specific surface receptors CD19 and CD20, the 
a

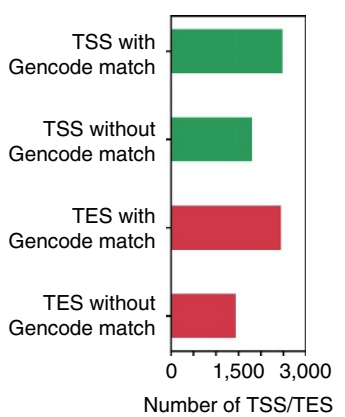

b

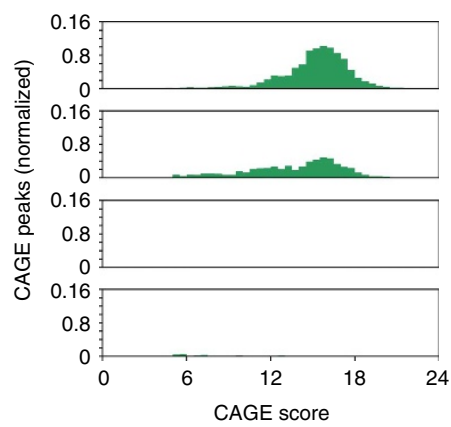

c

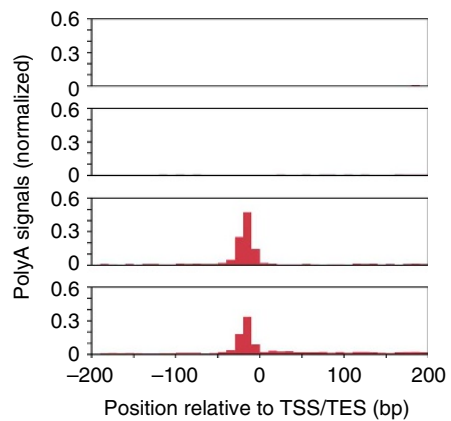

d

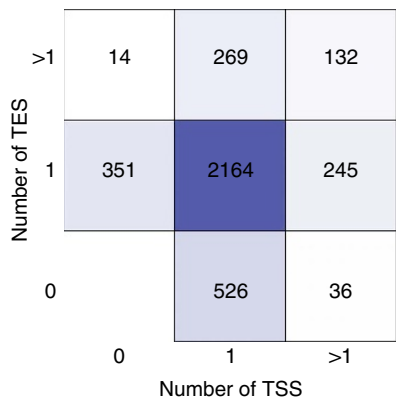

e

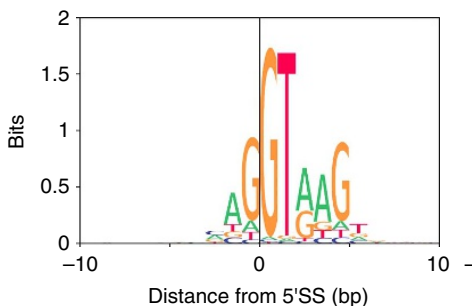

g

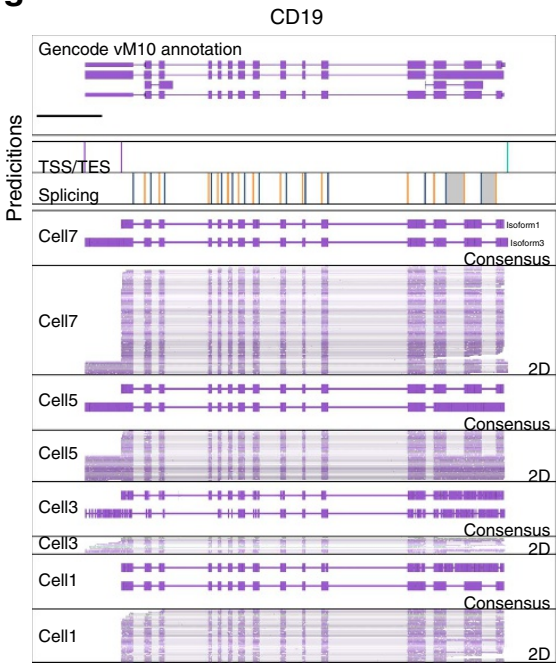

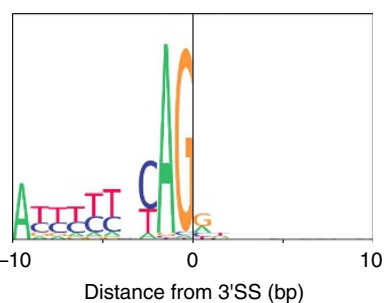

h

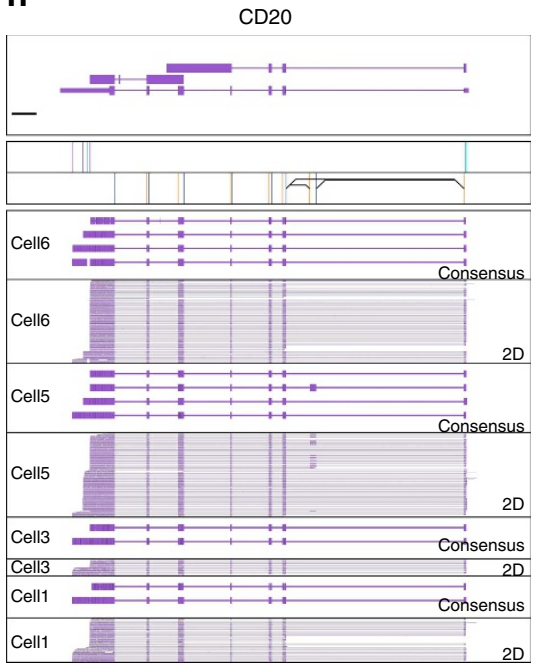

f

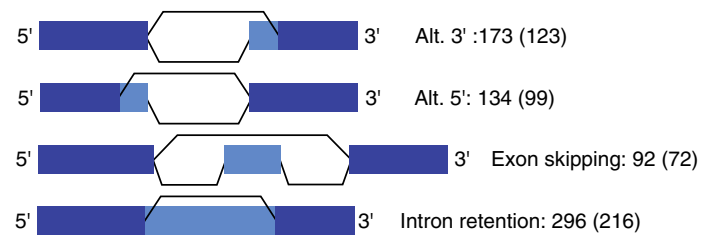

Figure 5 | Analysis of ONT RNAseq data identifies isoform features in mouse B1a cells. (a) TSSs and TESs predicted based on read data were separated into sites with or without GENCODE vM10 annotation matches. (b,c) TSSs/TESs with or without GENCODE matches were tested for FANTOM5 CAGE area enrichment (b) and polyA signals (c). (d) Overlap of TSSs and TESs with genes. Genes were sorted according to the number of TSSs and TESs they overlapped with. (e) Predicted base composition at $5^{\prime}$ and $3^{\prime}$ SS based on read data is shown as sequences logos. (f) Schematic for detection and corresponding number of detected alternative splice site combinations. Values in parentheses represent number of alternative splicing events validated using Illumina data. (g-i) Genome Browser view of CD19, CD20 and IGH gene loci as shown in Fig. 4. ONT 2D reads and consensus sequence alignments are shown for the indicated cells. Splice sites for the highly repetitive IGH locus were not considered for isoform grouping due to the difficulty of aligning reads unambigiously.

antibody heavy chain locus (IGH) (Fig. 5g-i), CD37 (Fig. 6), as well as $C D 2$ and $C D 79 b$, and $C D 45$ (Supplementary Fig. 5). We created consensus sequences of the isoforms at these gene loci in each B1a cell and found that across the individual B1a cells, isoforms derived from CD19 showed a combination of alternative TSSs and intron retention events. Isoforms derived from CD20, on the other hand, showed a combination of alternative TESs, as well as an exon skipping event including a previously unannotated exon. The IGH locus was even more complex, with canonical isoforms containing VDJ recombinations and the IGHM constant region exons. In addition, we observed isoforms containing the IGHM constant region exon but originating from
(1) abortive DJ recombinations (2) I-exon (3) miRNA loci in the IGHM Switch-region and (4) a J-segment. Finally, one isoform in cell 1 originated from the IGHM I-exon but contained the IGHD constant region exons. While IGH isoform diversity has been previously observed and has been known for a long time to be involved in class-switching ${ }^{43}$, the ability of ONT RNAseq to sequence full-length cDNA at the single cell level truly highlights and confirms the exceptional transcriptional diversity of the IGH locus.

The ability to sequence entire cDNA molecules from end to end presents an advantage over assembling transcript isoform using Illumina data. While assembling Illumina data using 


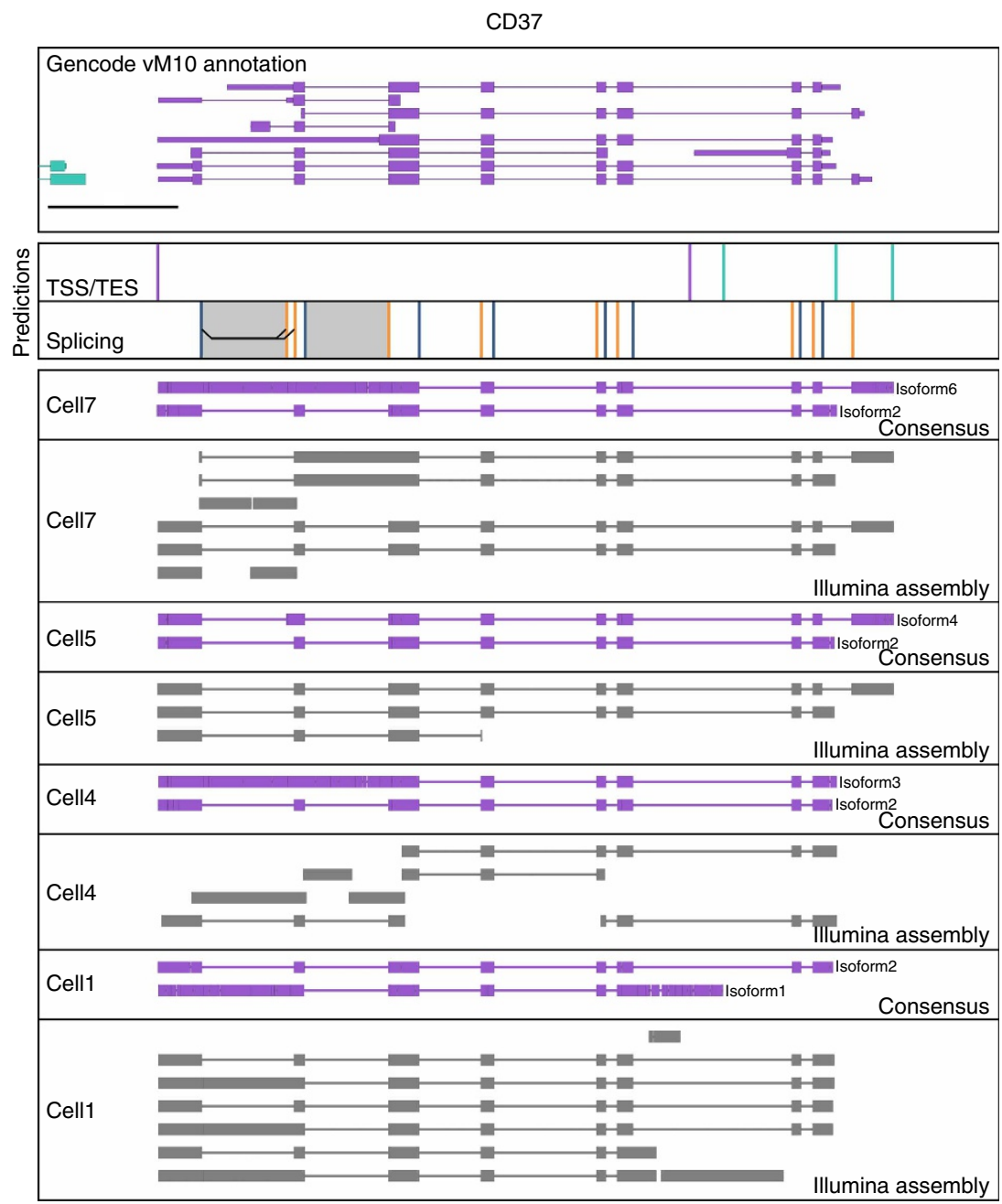

Figure 6 | Uncovering isoform diversity in B cell surface receptors. Genome Browser view of the CD37 is shown as in Fig. 4. In addition to isoform consensus derived from ONT 2D reads, contigs assembled from Illumina data using Trinity are shown in grey for the indicated cells.

Trinity ${ }^{44}$ is likely to succeed if a gene locus only expresses a single isoform, it appears to struggle with analysing multiple isoforms of a gene locus that contain multiple distant alternative features. For example, ONT RNAseq identified several distinct isoforms of the CD37 gene across the individual cells analysed. In most cases, when we assembled the Illumina data from individual cells, Trinity was either unable to form complete contigs or produced contigs that were not detected by ONT RNAseq (Fig. 6). The CD37 gene and its isoforms therefore highlight the strength of the ONT RNAseq approach to identify the diversity of complex isoforms beyond what is possible with either bulk or short reads technologies.

\section{Discussion}

The data we present here shows that RNAseq studies using the Oxford Nanopore Technologies MinION sequencer have the potential to redefine the level of information gathered by a single RNAseq experiment.

By benchmarking our experimental and computational pipelines on ONT MinION data derived from a mix of synthetic transcripts, we showed that our approach identifies the location of transcription start and end sites as well as splice sites in a genome. Furthermore, we have shown that these experimentally determined annotations can then be used by our Mandalorion pipeline to identify and quantify complex isoforms longer than $\sim 500 \mathrm{bp}$ in an otherwise largely transcript length independent manner. It is likely that if we use less stringent size selection methodologies during library preparation, we could capture transcripts $<500 \mathrm{bp}$ as well. Although we were only able to consistently identify the SIRV transcripts found among the high abundance groups, we expect that the less abundant transcripts could be identified using our pipeline by increasing the sequencing depth. Variation in the quantification of transcripts in the SIRV mix indicated that quantification might be improved by using Unique Molecular Identifiers (UMI) ${ }^{45}$ during cDNA amplification. However, UMI length would have to be at least $>30 \mathrm{bp}$ to be resolved unambiguously with the current error-rate of the ONT MinION. Introducing random nucleotides of this length during priming is likely to create short, unwanted PCR artifacts which would greatly increase the noise of the amplification reaction. Ultimately, until ONT sequencing accuracy improves, the Smartseq2 approach employed in this study is currently the best choice for UMI free library generation, as it has been shown by a comparison study to generate the smallest amount of PCR duplicates and the highest transcriptome coverage when comparing low input methodologies ${ }^{46-49}$.

By focusing on single-cells transcriptomes, we demonstrated the capability of sequencing read output and accuracy of ONT MinION sequencer. We showed that ONT RNAseq cannot only quantify known genes with a high correlation to Illumina RNAseq but, using the Mandalorion pipeline we developed, also 
annotate transcript features, thereby allowing us to identify and quantify complex, never before observed, isoforms. Using ONT RNAseq on only seven B1a cells, we identified thousands of unannotated transcription start and end sites which we then validated using FANTOM5 CAGE data and polyA signals, respectively. Furthermore, we identified 696 genes displaying alternative transcription start and end site usage, and 354 genes with alternative splicing events. Although not all alternative splicing events we detected were supported by single-cell Illumina data, the events that were not supported were of significantly lower coverage, indicating they might be closer to the detection limits in either technology (Supplementary Table 3). Combined with the relatively low Illumina sequencing depth per cell in our study, this suggests that larger Illumina depth might aid in the validation of individual events in future studies.

In addition to the identification of individual alternative events, the read length of the ONT MinION sequencer paired with our Mandalorion analysis pipeline enabled us to identify 169 genes expressing complex isoforms containing both alternative TSS/TES and splice sites. Interestingly, among the genes expressing these complex isoforms were surface receptors, including the very surface receptors distinguishing $\mathrm{B}$ cells from other immune cells. For example, we found that $C D 19, C D 20$ (Ms4a1), IGH, CD45 (B220 or Ptprc), CD2, CD79b and CD37 were expressed as multiple complex isoforms across the seven B1a cells. This indicates that the diversity of the surface receptors found on B-cells is not fully understood, which could have important implications on all facets of B cell biology. Our data suggest that we are currently only scratching the surface of the true transcriptional diversity of B1a cells. In the future, we aim to use the multiplexing strategy that we have developed to analyse hundreds of individual cells. This will make it possible to truly reconstitute the full transcriptome complexity of B1a and other cell types and will likely lead to discovery of additional subpopulations with distinct functional properties ${ }^{1}$. While we currently estimate the cost per cell at $\sim \$ 100-200$, this is likely to decrease considering the rapidly increasing throughput of the ONT MinION and the soon-to-be-released ONT PromethION sequencer.

Nanopore sequencing is still rapidly maturing and we believe that advancements in sequencing chemistries, nanopore design and analysis algorithms will vastly improve the technology and address the shortcomings of low read numbers and high error rates in the near future. Lower error-rates will, for example, allow us to improve the Mandalorion pipeline further by enabling the base accurate identification of TSS/TES and splice sites, instead of identifying $20 \mathrm{bp}$ bins for these features. Even with its current limitations, the data and analysis tools we present here demonstrate that ONT RNAseq is already a powerful addition to Illumina workflows and has the potential to revolutionize analysis of transcriptomes in the future. Finally, while the ONT MinION has not quite caught up with the very capable PacBio Sequel long read sequencer, it is only a fraction of its price $(\sim \$ 1,000$ versus $\$ 300,000)$. At this price, any molecular biology lab will be able to perform their own RNAseq experiments onsite, thereby increasing adoption of the single cell RNAseq approach and accelerating research.

\section{Methods}

FACS sorting of individual B cells. Mice were maintained in the UCSC vivarium according to protocols approved by UCSC's Institutional Animal Care and Use Committee (IACUC). Single murine Ter119 ${ }^{-} \mathrm{CD} 3^{-} \mathrm{CD} 4{ }^{-} \mathrm{CD} 8^{-} \mathrm{Gr} 1^{-} \mathrm{B} 220^{+}$ $\mathrm{IgM}^{+} \mathrm{CD} 1 \mathrm{~b}^{-} \mathrm{CD}^{+} \mathrm{B} 1 \mathrm{a}$ cells were isolated from wild-type $\mathrm{C} 57 \mathrm{Bl} / 6$ mice by lavage and incubated with fluorescently-labeled antibodies before sorting ${ }^{32}$. The following antibodies were purchased from Biolegend to stain B-cells: Ter119 (Biolegend catalogue number 116204; 1:100 dilution), CD3 (clone 145-2C11; 100304; 1:100), CD4 (GK1.5; 100404; 1:100), CD8a (53-6.7; 100704; 1:100),
streptavidin-cy5PE (405205; 1:400), B220 (RA3-6B2; 103224; 1:400), Gr1 (RB6$8 \mathrm{C} 5 ; 108404 ; 1: 200)$, IgM (RMM-1; 406514; 1:200), CD5 (53-7.3; 100614; 1:800) and CD11b (M1/70;101224; 1:1600). Cells were collected from the peritoneal cavity by lavage of $5 \mathrm{ml}$ PBS with $2 \%$ serum and $5 \mathrm{mM}$ EDTA. Similar to previously described protocols ${ }^{50-52}$, single-cell suspensions were incubated with the antibodies described above. Single cells were sorted into 96 well plates and directly placed into $4 \mu \mathrm{l}$ of Lysis Buffer- $0.1 \%$ Triton X-100, $0.2 \mu \mathrm{l}$ of SuperaseIn (Thermo), $1 \mu \mathrm{l}$ of oligodT primer (IDT), $1 \mu \mathrm{l}$ of dNTP (10 mM each) (NEB) - and frozen at $-80^{\circ} \mathrm{C}$.

Smartseq2 cDNA synthesis. Single cell lysate was reverse transcribed using Smartscribe Reverse Transcriptase (Clontech) in a $10 \mu$ l reaction including a Smartseq2 (ref. 34) TSO (Supplementary Table 1) according to manufacturer's instructions at $42^{\circ} \mathrm{C}$. The resulting cDNA was treated with $1 \mu \mathrm{l}$ of $1: 10$ dilutions of RNAse A (Thermofisher) and Lambda Exonuclease (NEB) for $30 \mathrm{~min}$ at $37^{\circ} \mathrm{C}$. A PCR amplification step using KAPA Hifi Readymix $2 \times$ (KAPA) step was performed incubating at $95^{\circ} \mathrm{C}$ for $3 \mathrm{~min}$, followed by 27 cycles of $\left(98^{\circ} \mathrm{C}\right.$ for $20 \mathrm{~s}$, $67^{\circ} \mathrm{C}$ for $15 \mathrm{~s}, 72^{\circ} \mathrm{C}$ for $4 \mathrm{~min}$ ), with a final extension at $72^{\circ} \mathrm{C}$ for $5 \mathrm{~min}$.

Illumina sequencing. The resulting full-length cDNA PCR product was treated with Tn5 enzyme ${ }^{53}$, which was loaded with Tn5ME-A/R and Tn5ME-B/R adaptors (Supplementary Table 1 ). The Tn5 product was then nick-translated and amplified for 13 cycles $\left(72{ }^{\circ} \mathrm{C}\right.$ for $6 \mathrm{~min}$, followed by $98^{\circ} \mathrm{C}$ for $30 \mathrm{~s}$ and 13 cycles of $\left(98^{\circ} \mathrm{C}\right.$ for $10 \mathrm{~s}, 63^{\circ} \mathrm{C}$ for $30 \mathrm{~s}, 72^{\circ} \mathrm{C}$ for $2 \mathrm{~min}$ ), with a final extension at $72^{\circ} \mathrm{C}$ for $5 \mathrm{~min}$ ) with KAPA Hifi Polymerase (KAPA) and Nextera Index Primers (Supplementary Table 1). Libraries were then size selected using a E-gel $2 \%$ EX (Thermo-Fisher) to a size range of 400-1,000 bp, and sequenced on an Illumina HiSeq2500 $2 \times 150$ run.

Nanopore sequencing. To achieve the $1 \mu \mathrm{g}$ of DNA needed for the Oxford Nanopore library prep, the full-length cDNA product was split into five aliquots and amplified for 13 cycles with KAPA Hifi Readymix $2 \times$ (KAPA) using the ISPCR or multiplex cellular index primers. The following reaction was incubated at $95^{\circ} \mathrm{C}$ for $3 \mathrm{~min}$, followed by 13 cycles of $\left(98^{\circ} \mathrm{C}\right.$ for $20 \mathrm{~s}, 67^{\circ} \mathrm{C}$ for $15 \mathrm{~s}, 72^{\circ} \mathrm{C}$ for $4 \mathrm{~min}$ ) with a final extension at $72^{\circ} \mathrm{C}$ for $5 \mathrm{~min}$. The single cDNA or multiplex product was further end-repaired and dA-tailed using NEBNext Ultra End Repair/ dA tailing mix (NEB), and adaptor ligated using the sequencing adaptors provided by ONT (HP Adaptor/Adaptor Mix). Ligation reaction was performed using Blunt/ TA ligase master mix (NEB). Reactions were then enriched using Dynabeads MyOne C1 Streptavidin (Life Technologies) to capture molecules that contain the HP Adaptor. Enriched libraries were then mixed with Fuel mix and Running buffer provided by ONT. Single-cell libraries were either sequenced solely on one (Cell1 and Cell2) or two (Cell3) separate MinION R7.3 flow cells and ran on the $48 \mathrm{~h} 2 \mathrm{D}$ protocol. For our multiplexing strategy, single R9.4 flow cells were used (Pool1: Cells4-7, Pool2: Lexogen libraries) and ran on the $48 \mathrm{~h} 2 \mathrm{D}$ protocol.

Data analysis. Illumina paired end $150 \mathrm{bp}$ reads in fastq format were quality and adaptor trimmed using trimmomatic ( $\mathrm{v} 0.33)^{54}$. The trimmed reads were aligned using STAR (v2.4) ${ }^{35}$ to the mouse genome (Sequence: GRCm38, Annotation: Gencode vM10 (ref. 41)). Illumina reads were assembled for each cell separately using the Trinity (v2.2.0) ${ }^{44}$ set of tools.

ONT reads were processed using the Metrichor cloud platform 2D workflow. For R7.3 runs, both reads that passed or failed Metrichor quality cutoffs were retained. For R9.4 runs, reads that failed Metrichor quality cutoffs were discarded as they also failed our alignment criteria. Fast 5 files generated by Metrichor were converted into fastq and fasta formats using poretools (v0.5.1) ${ }^{55}$.

For demultiplexing, index-sequences were aligned to the reads using BLAT with parameters: -noHead -stepSize $=1$-minScore $=20$-minIdentity $=20$. Reads for which index-sequences could be identified were trimmed and assigned to the respective libraries. Next, for multiplexed and non-multiplexed reads alike, ISPCR adaptor sequences were identified and trimmed using Levenshtein distances. Reads for which ISPCR adaptors could be identified and trimmed were marked but all reads, trimmed or not, were aligned to the mouse genome (GRCm38) using BLAT $(\mathrm{v} 35 \times 1)^{37}$ with parameters: - stepSize $=5$-repMatch $=2253$ -

minScore $=100$-minIdentity $=50$-maxIntron $=2,000,000$. Alignments were filtered for a single alignment per read. This filtering process involved three steps: (i) the highest scoring alignment for each read is identified, alignment scores within $2 \%$ of each other were treated as ties, (ii) in case of ties the alignment with largest number of gaps is selected (this selects against alignment to unspliced pseudogenes) and (iii) if the best alignment of a read has a ratio of aligned bases to read bases $\leq 0.6$ the read and its alignment are discarded.

Gene Expression for ONT and Illumina RNAseq was analysed using custom scripts. For each gene, the number of reads overlapping with its exons was counted, normalized to total number of aligned reads in a library and reported as Reads Per Gene per 10,000 reads (RPG10K). Genes were counted as expressed if they had a RPG10K value $>0$. RPG can be calculated as: RPG10k= (total \# of reads aligned to gene exon/total \# of reads aligned in sample) $\times 10,000$.

For the detection transcription start and end sites we limited our analysis to reads for which we detected and trimmed ISPCR adaptor before read alignment. 
We then identified positions in the genome at which at least two alignments of these complete reads ended. We then further restricted our analysis by only considering positions with a median and 75th percentile of the number of clipped (unaligned) read bases between $6-15$ and $\leq 20$, respectively. This number of clipped (unaligned) bases corresponds to the length of bases contained in ISPCR TSO and oligodT primers that were not trimmed. We then placed a $20 \mathrm{bp}$ bin around these positions to include the highest number of read alignment ends possible.

To filter false positive bins caused by incomplete read alignments in highly expressed genes bins were only considered as containing true TSS/TES if they met the following conditions:

(i) The total number of read alignment ends in the bins had to be $\geq 2 \%$ of the total number of reads in the next 50 read covered bases. (ii) The candidate site had to be at least 60 read covered bases away from the next closest TSS/TES.

By only counting bases covered by read alignments we didn't take non-covered introns into account which would skew our analysis. Next, in order to distinguish TSS and TES bins, we calculated median Levenshtein distances of the unaligned bases at all read alignment ends in a bin to nucleotides present in TSO (ATGG) or the OligodT (TTTT) primer. If the median Levenshtein of a bin to ATGG was $\leq 2$ it was declared a TSS. If the median Levenshtein of a bin to TTTT was $\leq 2$ it was declared a TES.

To assess Fantom 5 (ref. 42) CAGE scores we downloaded combined CAGE data (mm9.cage_peak_phaseland2combined_coord.bed.gz), converted the data to $\mathrm{mm} 10$ coordinates using https://genome.ucsc.edu/cgi-bin/hgLiftOver ${ }^{56}$ and investigated direct overlap between TSS/TES and CAGE peaks. We considered TSS/TES and CAGE peaks to be overlapping if they were within $10 \mathrm{bp}$ of each other. To assess polyA enrichment in TSS/TES, we extracted genomic sequences up- and downstream of these sites and looked for identical string matches to 'AATAAA' and 'ATTAAA'.

To identify 20 bp bins as splice sites only ONT 2 D reads with a ratio of aligned bases/read bases of $>0.9$ were analysed. We then identified positions in the genome at which at least two read alignments of these reads opened or closed an alignment gap larger than $50 \mathrm{bp}$.

The $20 \mathrm{bp}$ bins surrounding these positions were considered as containing a splice site if the following conditions are met:

(i) The number of of reads opening or closing an alignment gap in the bin was at least $2 \%$ of the total number of reads in the preceding $\left(5^{\prime}\right)$ or subsequent $\left(3^{\prime}\right) 40$ read covered bases. (ii) Not closer than $30 \mathrm{bp}$ to another splice site. The directionality of the splice site bin containing either $5^{\prime}$ or $3^{\prime}$ status was based on the direction of the majority of reads containing the splice site.

To detect alternative splice sites, we counted how often $5^{\prime}$ and $3^{\prime}$ splice sites were spliced together in ONT $2 \mathrm{D}$ reads with aligned bases/read bases ratio of $>0.85$. A $5^{\prime}->3^{\prime}$ combination had to be present in at least two reads to be considered. We scored alternative splice site usage if the same $5^{\prime}$ splice site was spliced into two different $3^{\prime}$ splice sites or vice versa. To detect intron retentions, we identified areas between $5^{\prime}$ and $3^{\prime}$ splice sites that were covered to at least $70 \%$ by at least one ONT $2 \mathrm{D}$ read.

We detected isoforms by grouping reads according the TSS/TES and alternative splice sites they contained. ONT read alignment ends found within $60 \mathrm{bp}$ of a TSS and a TES were sorted based on which alternative splice sites it contained. Isoforms that contained at least $1 \%$ of all reads at a gene locus were retained. All the reads in these retained isoform groups were used to create consensus reads using $\mathrm{POA}^{39}$. In short, fasta files containing all read sequence are passed to POA, which generates a consensus of the reads by creating a multiple sequence alignment of the reads in the form of a partially ordered graph. The program then returns the most heavilyweighted path as the consensus of the reads. The consensus reads are then aligned to genome using BLAT parameters: - stepSize $=5$-repMatch $=2253$ -

minscore $=10$-minIdentity $=10$. There was however, one exception regarding the highly complex variable regions derived from the IGH transcripts which were first aligned with IgBlast ${ }^{57}$ and then with BLAT. IgBlast alignment coordinates were converted to genome coordinates and BLAT and IgBlast portions of the read alignments were merged.

We used the 'chi2_contingency' function in the scipy.stats ${ }^{58}$ package to implement the Chi2 contingency test to detect differential expression of complex isoforms between cells. Multiple testing holm-sidak correction was performed with the 'statsmodels.sandbox.stats.multicomp" 59 package.

All data analysis and visualization was performed in python ${ }^{60}$ using the numpy/ scipy/matplotlib ${ }^{58,61,62}$ packages.

Code availability. All scripts, including the Mandalorion pipeline, are available on request and are available at GitHub at https://github.com/christopher-vollmers/ Mandalorion.

Data availability. Illumina and ONT sequencing reads were uploaded to the SRA under accession number SRP082530. The rest of the data that support the conclusions of this study are available from the corresponding author upon request.

\section{References}

1. Wu, A. R. et al. Quantitative assessment of single-cell RNA-sequencing methods. Nat. Methods 11, 41-46 (2014).
2. Treutlein, B. et al. Reconstructing lineage hierarchies of the distal lung epithelium using single-cell RNA-seq. Nature 509, 371-375 (2014).

3. Shalek, A. K. et al. Single-cell transcriptomics reveals bimodality in expression and splicing in immune cells. Nature 498, 236-240 (2013).

4. Welch, J. D., Hu, Y. \& Prins, J. F. Robust detection of alternative splicing in a population of single cells. Nucleic Acids Res. 44, e73 (2016).

5. Stamm, S. et al. Function of alternative splicing. Gene 344, 1-20 (2005).

6. Sugnet, C. W., Kent, W. J., Ares, Jr M. \& Haussler, D. Transcriptome and genome conservation of alternative splicing events in humans and mice. Pac. Symp. Biocomput. 9, 66-77 (2004).

7. Modrek, B. \& Lee, C. A genomic view of alternative splicing. Nat. Genet. 30, 13-19 (2002).

8. Wang, E. T. et al. Alternative isoform regulation in human tissue transcriptomes. Nature 456, 470-476 (2008).

9. Krawczak, M., Reiss, J. \& Cooper, D. N. The mutational spectrum of single base-pair substitutions in mRNA splice junctions of human genes: causes and consequences. Hum. Genet. 90, 41-54 (1992).

10. Brooks, A. N. et al. A pan-cancer analysis of transcriptome changes associated with somatic mutations in U2AF1 reveals commonly altered splicing events. PLoS ONE 9, e87361 (2014).

11. Salzberg, S. L. et al. GAGE: a critical evaluation of genome assemblies and assembly algorithms. Genome Res. 22, 557-567 (2012).

12. Tilgner, H. et al. Comprehensive transcriptome analysis using synthetic longread sequencing reveals molecular co-association of distant splicing events. Nat. Biotechnol. 33, 736-742 (2015).

13. Sharon, D., Tilgner, H., Grubert, F. \& Snyder, M. A single-molecule long-read survey of the human transcriptome. Nat. Biotechnol. 31, 1009-1014 (2013).

14. Treutlein, B., Gokce, O., Quake, S. R. \& Südhof, T. C. Cartography of neurexin alternative splicing mapped by single-molecule long-read mRNA sequencing. Proc. Natl Acad. Sci. USA 111, E1291-E1299 (2014).

15. Vollmers, C., Penland, L., Kanbar, J. N. \& Quake, S. R. Novel exons and splice variants in the human antibody heavy chain identified by single cell and single molecule sequencing. PLOS ONE 10, e0117050 (2015).

16. Oikonomopoulos, S., Wang, Y. C., Djambazian, H., Badescu, D. \& Ragoussis, J. Benchmarking of the Oxford Nanopore MinION sequencing for quantitative and qualitative assessment of cDNA populations. Sci. Rep. 6, 31602 (2016).

17. Weirather, J. L. et al. Comprehensive comparison of Pacific Biosciences and Oxford Nanopore Technologies and their applications to transcriptome analysis. F1000Res. 6 (2017).

18. Macaulay, I. C. et al. G\&T-seq: parallel sequencing of single-cell genomes and transcriptomes. Nat. Methods 12, 519-522 (2015).

19. Karlsson, K. \& Linnarsson, S. Single-cell mRNA isoform diversity in the mouse brain. BMC Genomics 18, 126 (2017).

20. Graf, T. \& Stadtfeld, M. Heterogeneity of embryonic and adult stem cells. Cell Stem Cell 3, 480-483 (2008).

21. Irish, J. M., Kotecha, N. \& Nolan, G. P. Mapping normal and cancer cell signalling networks: towards single-cell proteomics. Nat. Rev. Cancer 6, 146-155 (2006).

22. Warren, L., Bryder, D., Weissman, I. L. \& Quake, S. R. Transcription factor profiling in individual hematopoietic progenitors by digital RT-PCR. Proc. Natl Acad. Sci. USA 103, 17807-17812 (2006).

23. Cornelison, D. D. \& Wold, B. J. Single-cell analysis of regulatory gene expression in quiescent and activated mouse skeletal muscle satellite cells. Dev. Biol. 191, 270-283 (1997).

24. Raj, A., van den Bogaard, P., Rifkin, S. A., van Oudenaarden, A. \& Tyagi, S. Imaging individual mRNA molecules using multiple singly labeled probes. Nat. Methods 5, 877-879 (2008).

25. Tang, F., Lao, K. \& Surani, M. A. Development and applications of single-cell transcriptome analysis. Nat. Methods 8, S6-11 (2011).

26. Femino, A. M., Fay, F. S., Fogarty, K. \& Singer, R. H. Visualization of single RNA transcripts in situ. Science 280, 585-590 (1998).

27. Cherf, G. M. et al. Automated forward and reverse ratcheting of DNA in a nanopore at 5-Å precision. Nat. Biotechnol. 30, 344-348 (2012).

28. Hargreaves, A. D. \& Mulley, J. F. Assessing the utility of the Oxford Nanopore MinION for snake venom gland cDNA sequencing. Peer J. 3, el441 (2015).

29. Kilianski, A. et al. Bacterial and viral identification and differentiation by amplicon sequencing on the MinION nanopore sequencer. Gigascience 4, 12 (2015).

30. Jain, M. et al. Improved data analysis for the MinION nanopore sequencer. Nat. Methods 12, 351-356 (2015).

31. Bolisetty, M. T., Rajadinakaran, G. \& Graveley, B. R. Determining exon connectivity in complex mRNAs by nanopore sequencing. Genome Biol. 16, 204 (2015).

32. Beaudin, A. E. et al. A transient developmental hematopoietic stem cell gives rise to innate-like B and T cells. Cell Stem Cell 19, 768-783 (2016).

33. Beaudin, A. E. \& Forsberg, E. C. To B1a or not to B1a: do hematopoietic stem cells contribute to tissue-resident immune cells? Blood 128, 2765-2769 (2016). 
34. Picelli, S. et al. Smart-seq2 for sensitive full-length transcriptome profiling in single cells. Nat. Methods 10, 1096-1098 (2013).

35. Dobin, A. et al. STAR: ultrafast universal RNA-seq aligner. Bioinformatics 29, 15-21 (2013).

36. Trapnell, C. et al. Transcript assembly and quantification by RNA-Seq reveals unannotated transcripts and isoform switching during cell differentiation. Nat. Biotechnol. 28, 511-515 (2010).

37. Kent, W. J. BLAT-the BLAST-like alignment tool. Genome Res. 12, 656-664 (2002).

38. Macosko, E. Z. et al. Highly parallel genome-wide expression profiling of individual cells using nanoliter droplets. Cell 161, 1202-1214 (2015)

39. Lee, C., Grasso, C. \& Sharlow, M. F. Multiple sequence alignment using partial order graphs. Bioinformatics 18, 452-464 (2002).

40. Stanke, M., Steinkamp, R., Waack, S. \& Morgenstern, B. AUGUSTUS: a web server for gene finding in eukaryotes. Nucleic Acids Res. 32, W309-W312 (2004).

41. Mudge, J. M. \& Harrow, J. Creating reference gene annotation for the mouse C57BL6/J genome assembly. Mamm. Genome 26, 366-378 (2015).

42. FANTOM Consortium and the RIKEN PMI and CLST (DGT) et al. A promoter-level mammalian expression atlas. Nature 507, 462-470 (2014).

43. Islam, K. B., Baskin, B., Christensson, B., Hammarström, L. \& Smith, C. I. In vivo expression of human immunoglobulin germ-line mRNA in normal and in immunodeficient individuals. Clin. Exp. Immunol. 95, 3-9 (1994).

44. Grabherr, M. G. et al. Full-length transcriptome assembly from RNA-Seq data without a reference genome. Nat. Biotechnol. 29, 644-652 (2011).

45. Islam, S. et al. Quantitative single-cell RNA-seq with unique molecular identifiers. Nat. Methods 11, 163-166 (2014).

46. Bhargava, V., Head, S. R., Ordoukhanian, P., Mercola, M. \& Subramaniam, S. Technical variations in low-input RNA-seq methodologies. Sci. Rep. 4, 3678 (2014).

47. Svensson, V. et al. Power analysis of single-cell RNA-sequencing experiments. Nat. Methods 14, 381-387 (2017).

48. Dueck, H. R. et al. Assessing characteristics of RNA amplification methods for single cell RNA sequencing. BMC Genomics 17, 966 (2016).

49. Ziegenhain, C. et al. Comparative Analysis of Single-Cell RNA Sequencing Methods. Mol. Cell 65, 631-643.e4 (2017)

50. Ugarte, F. et al. Progressive chromatin condensation and H3K9 methylation regulate the differentiation of embryonic and hematopoietic stem cells. Stem Cell Rep. 5, 728-740 (2015).

51. Smith-Berdan, S., Nguyen, A., Hong, M. A. \& Forsberg, E. C. ROBO4-mediated vascular integrity regulates the directionality of hematopoietic stem cell trafficking. Stem Cell Rep. 4, 255-268 (2015).

52. Beaudin, A. E., Boyer, S. W. \& Forsberg, E. C. Flk2/Flt3 promotes both myeloid and lymphoid development by expanding non-self-renewing multipotent hematopoietic progenitor cells. Exp. Hematol. 42, 218-229.e4 (2014).

53. Picelli, S. et al. Tn5 transposase and tagmentation procedures for massively scaled sequencing projects. Genome Res. 24, 2033-2040 (2014).

54. Bolger, A. M., Lohse, M. \& Usadel, B. Trimmomatic: a flexible trimmer for Illumina sequence data. Bioinformatics 30, 2114-2120 (2014).

55. Loman, N. J. \& Quinlan, A. R. Poretools: a toolkit for analyzing nanopore sequence data. Bioinformatics 30, 3399-3401 (2014).

56. Kent, W. J. et al. The human genome browser at UCSC. Genome Res. 12, 996-1006 (2002).

57. Ye, J., Ma, N., Madden, T. L. \& Ostell, J. M. IgBLAST: an immunoglobulin variable domain sequence analysis tool. Nucleic Acids Res. 41, W34-W40 (2013).

58. Jones, E. et al. \{SciPy\}: Open source scientific tools for \{Python\} http:// www.scipy.org/ (2001)

59. Seabold, S. \& Perktold, J. Statsmodels: Econometric and Statistical Modeling with Python. Proceedings of the 9th Python in Science Conference, 57-61 (2010).
60. Oliphant, T. E. Python for scientific computing. Comput. Sci. Eng. 9, 10-20 (2007). 61. van der Walt, S., Colbert, S. C. \& Varoquaux, G. The NumPy Array:

A Structure for Efficient Numerical Computation. Comput. Sci. Eng. 13, 22-30 (2011).

62. Hunter, J. D. Matplotlib: A 2D Graphics Environment. Comput. Sci. Eng. 9, 90-95 (2007)

\section{Acknowledgements}

Research reported in this publication was supported by NIH/NHGRI awards HG006321 (M.A.) and HG007827 (M.A.); by the NIH/NIDDK award R01DK100917 (E.C.F.); by Alex's Lemonade Stand Foundation Innovation Award (E.C.F.); by the American Asthma Foundation (E.C.F.); by CIRM Training grant TG2-01157 to A.E.B.; by NHLBI K01HL130753-01A1 to A.E.B.; by CIRM Shared Stem Cell Facilities CL1-00506 and CIRM Major Facilities FA1-00617-1 awards to UCSC. E.C.F. is the recipient of a California Institute for Regenerative Medicine (CIRM) New Faculty Award RN1-00540 and an American Cancer Society Research Scholar Award RSG-13-193-01-DDC. A.B. and C.C. are funded by the NHGRI/NIH training grant 1T32HG008345-01.

\section{Author contributions}

C.V., M.A. and E.C.F. conceived of the research. C.V. directed the research. C.V., A.B. and C.C. analysed the data. C.V. and A.B. wrote the paper. A.B., A.E.B., T.P., M.J. and H.E.O. designed and performed experiments. R.M.D. contributed materials. All authors edited the manuscript.

\section{Additional information}

Supplementary Information accompanies this paper at http://www.nature.com/ naturecommunications

Competing interests: M.A. is a paid consultant to Oxford Nanopore Technologies (Oxford, UK). The remaining authors declare no competing financial interests.

Reprints and permission information is available online at http://npg.nature.com/ reprintsandpermissions/

How to cite this article: Byrne, A. et al. Nanopore long-read RNAseq reveals widespread transcriptional variation among the surface receptors of individual B cells. Nat. Commun. 8, 16027 doi: 10.1038/ncomms16027 (2017)

Publisher's note: Springer Nature remains neutral with regard to jurisdictional claims in published maps and institutional affiliations.

Open Access This article is licensed under a Creative Commons Attribution 4.0 International License, which permits use, sharing, adaptation, distribution and reproduction in any medium or format, as long as you give appropriate credit to the original author(s) and the source, provide a link to the Creative Commons license, and indicate if changes were made. The images or other third party material in this article are included in the article's Creative Commons license, unless indicated otherwise in a credit line to the material. If material is not included in the article's Creative Commons license and your intended use is not permitted by statutory regulation or exceeds the permitted use, you will need to obtain permission directly from the copyright holder. To view a copy of this license, visit http://creativecommons.org/ licenses/by/4.0/

C) The Author(s) 2017 